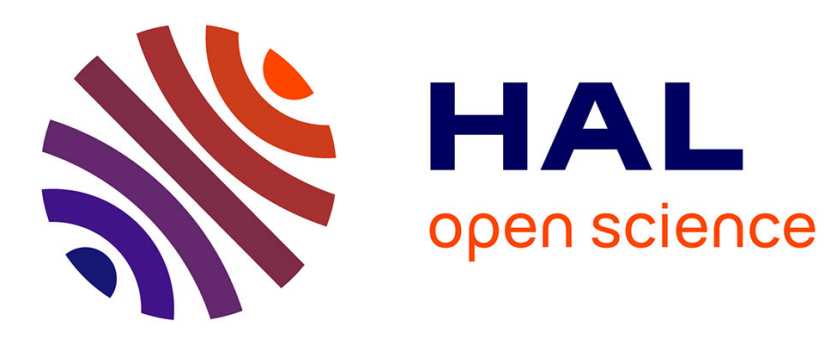

\title{
Dependability Evaluation of Cooperative Backup Strategies for Mobile Devices
}

\author{
Ludovic Courtès, Ossama Hamouda, Mohamed Kaâniche, Marc-Olivier
} Killijian, David Powell

\section{- To cite this version:}

Ludovic Courtès, Ossama Hamouda, Mohamed Kaâniche, Marc-Olivier Killijian, David Powell. Dependability Evaluation of Cooperative Backup Strategies for Mobile Devices. 13th Pacific Rim International Symposium on Dependable Computing (PRDC 2007), Dec 2007, Melbourne, Australia. pp.139 - 146, 10.1109/PRDC.2007.21 . hal-00851766

\section{HAL Id: hal-00851766 https://hal.science/hal-00851766}

Submitted on 6 Oct 2015

HAL is a multi-disciplinary open access archive for the deposit and dissemination of scientific research documents, whether they are published or not. The documents may come from teaching and research institutions in France or abroad, or from public or private research centers.
L'archive ouverte pluridisciplinaire HAL, est destinée au dépôt et à la diffusion de documents scientifiques de niveau recherche, publiés ou non, émanant des établissements d'enseignement et de recherche français ou étrangers, des laboratoires publics ou privés. 
Dependability Evaluation of Cooperative Backup Strategies for Mobile Devices

\author{
Ludovic Courtès, Ossama Hamouda, \\ Mohamed Kaâniche, Marc-Olivier Killijian, David Powell \\ LAAS-CNRS, University of Toulouse, France
}

\begin{abstract}
Mobile devices (e.g., laptops, PDAs, cell phones) are increasingly relied on but are used in contexts that put them at risk of physical damage, loss or theft. This paper discusses the dependability evaluation of a cooperative backup service for mobile devices. Participating devices leverage encounters with other devices to temporarily replicate critical data. Permanent backups are created when the participating devices are able to access the fixed infrastructure. Several data replication and scattering strategies are presented, including the use of erasure codes. A methodology to model and evaluate them using Petri nets and Markov chains is described. We demonstrate that our cooperative backup service decreases the probability of data loss by a factor up to the ad hoc to Internet connectivity ratio.
\end{abstract}

\section{Introduction}

Mobile devices (e.g., laptops, PDAs, cell phones) are increasingly relied on but are used in contexts that put them at risk of physical damage, loss or theft. However, fault-tolerance mechanisms available for these devices often suffer from shortcomings. For instance, data "synchronization" mechanisms, which allow one to replicate a mobile device's data on a desktop machine, usually require the desktop machine to be either physically accessible or reachable via the Internet. Use of third-party backup servers typically also requires access to some network infrastructure.

We aim to address these issues by providing a cooperative backup service, called MoSAIC [3, 12]. Our approach seeks to apply the peer-to-peer data storage and backup paradigm that has been successful on the Internet [7, 14] to data backup among communicating devices. Participating mobile devices share storage resources. When devices encounter one another, they may

\footnotetext{
* This work was partially supported by the MoSAIC project (ACI S\&I, French national program for Security and Informatics; see http://www.laas.fr/mosaic/), the Hidenets project (EU-ISTFP6-26979), and the ReSIST network (EU-IST-FP6-26764).
}

exchange critical data and store the data received from the peer device; eventually, when a participating device gains access to the Internet, it forwards data stored on behalf of other nodes to an Internet-accessible store.

Various replication and data scattering algorithms may be used to implement the cooperative backup service. Replication may be handled by creating full copies of individual data items (we refer to this as simple replication) or by more sophisticated erasure coding techniques. Choosing between these techniques implies a tradeoff between storage efficiency and data confidentiality [3]. This tradeoff can be done beforehand and is well understood; however, its impact on data dependability, particularly in our scenario, is unclear. The analytical evaluation presented in this paper aims to clarify this.

We analyze the fault-tolerance gain provided by MoSAIC as a function of (i) the various environmental parameters (frequency of Internet access, device encounter rate, node failure rate) and (ii) different replication strategies. Our approach is based on model-based evaluation, which is well suited to support design tradeoff studies and to analyze the impact of several parameters of the design and the environment from the dependability and performance points of view.

The analysis presented in this paper has two main goals. First, it should help us determine under what circumstances MoSAIC is the most beneficial, compared to solutions that do not replicate data in the ad hoc domain. Second, it should help us choose among different replication strategies, depending on a given scenario's parameters and user preferences (e.g., target data availability, confidentiality requirements).

The work presented here builds on our previous work on the design of a cooperative backup service for mobile devices $[3,5,12]$. Its major contribution lies in the dependability evaluation of data replication and scattering strategies using analytical methods, taking into account a variety of influential parameters. It differs substantially from earlier evaluation work by other authors due to the entirely novel characteristics of the system and environment modelled (see Section 5). 
Section 2 provides an overview of MoSAIC and background information on erasure codes. Section 3 describes our methodology. Section 4 summarizes the results obtained and discusses their impact on the design of the cooperative backup service. Section 5 presents related work. Finally, Section 6 concludes on our findings and depicts future research directions.

\section{Background}

This section gives an overview of MoSAIC and provides background information about erasure codes.

\subsection{MoSAIC Overview}

Our cooperative backup service, which we call MoSAIC, can leverage (i) excess storage resources available on mobile devices and (ii) short-range, high-bandwidth, and relatively energy-efficient wireless communications (Bluetooth, Zigbee, or Wi-Fi). The aim is to improve long-term availability of data produced by mobile devices. The idea is borrowed from peer-to-peer cooperative services: participating devices offer storage resources and doing so allows them to benefit from the resources provided by other devices in order to replicate their data [12]. Participating devices discover other devices in their vicinity and communicate through single-hop connections, thereby limiting interactions to small physical regions.

Anyone is free to participate in the service and, therefore, participants have no prior trust relationship. We use the term contributor when referring to a device acting as a storage provider; we use the term data owner when referring to a "client" device, i.e., one that uses storage provided by the contributors to replicate its data. All devices may play both the owner and the contributor role.

Since participating devices are mutually distrustful, the storage layer of the backup service must guarantee confidentiality through encryption [3]. Care is also taken to minimize attacks to user privacy and to protect against denial-of-service attacks such as refusal to cooperate or flooding [5]. In this paper, we do not focus on these issues, but rather, we explore possible strategies to maximize the chances of being able to restore the user's data in the presence of faulty participants, be they malicious or not.

When out of reach of Internet access and network infrastructure, devices may meet and spontaneously form ad hoc networks that they can use to back-up data. Since it would be unrealistic to rely on chance encounters between devices for recovery, we require contributing devices to eventually send data stored on behalf of other devices to an Internet-based store accessible by the data owners [3, 12].
MoSAIC's approach to cooperative backup bears some similarity with earlier work on cooperative data storage $[2,10]$ and caching for mobile devices [18, 24]. However, it differs from them in several ways. First, unlike typical distributed file systems access patterns, data that is backed up is produced by a single device and may usually not be accessed by other devices. Second, unlike most caching strategies, our approach does not seek to improve locality of data replicas: instead we expect replicas to propagate to the Internet-based store, much like packets in a delay-tolerant network (DTN) [25].

\subsection{Erasure Codes}

Erasure coding algorithms have been studied extensively $[15,17,21,22,23]$. Here we do not focus on the algorithms themselves but on their properties. A commonly accepted definition of erasure coding is the following $[15,23]$ :

- Given a $k$-symbol input datum, an erasure coding algorithm produces $n \geq k$ fragments.

- $m$ fragments are necessary and sufficient to recover the original datum, where $k \leq m \leq n$. When $m=k$, the erasure code algorithm is said to be optimal [22].

Although not all erasure coding algorithms are optimal (many of them are near-optimal [22]), we will assume in the sequel the use of an optimal erasure code where $m=k$. By convention, we note $(n, k)$ such an optimal erasure code [23].

When all $k$ fragments are stored on different devices, an optimal erasure code allows $n-k$ failures (or erasures) to be tolerated (beside that of the primary replica). The storage cost (or stretch factor) for an optimal erasure code is $\frac{n}{k}$ (the inverse ratio $\frac{k}{n}$ is often called the rate of an erasure code). To tolerate a number of erasures $f$, we need $n=k+f$, so the storage cost is $1+{ }^{f} / k$. Therefore, erasure coding (with $k \geq 2$ ) is more storage-efficient than simple replication $(k=1)$. For instance, $(2,1)$ and $(3,2)$ erasure codes both tolerate one failure, but the former requires twice as much storage as the original data while the latter only requires 1.5 times as much.

When all $k$ fragments are distributed to different devices belonging to different non-colluding users (or under different administrative domains), erasure codes can be regarded as a means for improving data confidentiality: to access the data, an attacker must have control over $k$ contributing devices instead of just one when simple replication is used [8]. This effectively raises the bar for confidentiality attacks and may usefully complement ciphering techniques used at other layers. Similar concerns are addressed by generalized threshold schemes where, in addition to the definition above, less than $p \leq k$ 
fragments convey no information about the original data, from an information-theoretic viewpoint [9].

\section{Methodology}

In this section, we present the approach we have taken to model our cooperative backup service, and the dependability measures we made.

\subsection{System Characteristics}

The backup service that we model is characterized by its replication and scattering strategy, and the device-to-device and device-to-Internet backup opportunities.

\subsubsection{Replication Strategy}

We consider the case of a data owner that needs to replicate a single data item (generalization to more than one data item is straightforward). We consider that the owner follows a pre-defined replication strategy, using $(n, k)$ erasure coding, where $n$ is decided off-line, a priori, and where the owner distributes one and only one fragment to each encountered contributor. When $k=1$, the strategy corresponds to simple replication. In practice, the choice of $n$ and $k$ could be made as a function of the scenario's characteristics and the user's dependability and confidentiality requirements.

This replication strategy favors confidentiality over data dependability: only one fragment is given to each contributor encountered ${ }^{1}$, at the risk of being unable to distribute all the fragments in the end (for instance, because not enough contributors become available). An alternative strategy that favors data dependability over confidentiality consists in providing a contributor with as many fragments as possible while it is reachable.

We consider a static replication strategy. In particular, we suppose that owners are not aware of the failures of contributors storing data on their behalf. Thus, owners cannot, for instance, decide to create more replicas when previously encountered contributors have failed. This assumption is realistic under most of the scenarios envisaged: first, detection of the failure of a participating device would be hard to achieve in a mobile context where nodes continuously come and go, and second, since devices are mobile, they are likely to be out of reach at the time the contributor fails.

\subsubsection{Backup Opportunities}

We consider that every encounter between devices offers a backup opportunity. Specifically, every device encoun-

\footnotetext{
${ }^{1}$ According to this policy, a contributor encountered more than once will only be given a fragment the first time it is encountered.
}

tered is considered to be a contributor that unconditionally accepts storage requests from the data owner. Data owners unconditionally send one data fragment to each contributor encountered. Note that scenarios in which not all encounters offer backup opportunities (e.g., with contributors refusing to cooperate) can be simply modeled by introducing an opportunity/encounter ratio as an additional parameter.

We consider that Internet connection is only exploited when it is cheap and provides a high bandwidth. Thus, whenever a node gains Internet access, we assume that it transfers all the data fragments it currently stores on behalf of other nodes.

\subsection{Modeling Approach}

Analytical model-based techniques are commonly used to support dependability evaluation studies. They allow one to obtain mathematical expressions of the relevant measures, which can then be explored to easily identify trends and to carry out sensitivity analysis. When using analytical techniques, the system must be described at a high level of abstraction. Simplifying assumptions are generally needed to obtain tractable models. Although simulation can be used to describe the system at a more detailed level, it is more costly in terms of the processing time needed to obtain accurate and statistically significant quantitative results.

Markov chains and generalized stochastic Petri nets (GSPNs) are commonly used to perform dependability evaluation studies and sensitivity analyses aimed at identifying parameters having the most significant impact on the measures. The corresponding models are based on the assumption that all the underlying stochastic processes are described by exponential distributions. Although such an assumption may not faithfully reflect reality, the results obtained from the models give preliminary indications about the expected behaviors and trends that can be observed. More accurate results can be obtained considering more general distributions, using for example the "stages method" [6] or non Markovian models. However, in this paper, we assume that all stochastic processes are exponentially distributed and we use GSPNs for the construction of Markov models [16].

\subsection{GSPN and Markov Models}

Figure 1 presents the GSPN model of MoSAIC using an $(n, k)$ erasure coding algorithm. The model focuses on the mobile ad hoc part of the cooperative backup service, purposefully ignoring issues related to the implementation of the Internet-side functionalities. A data fragment is considered "safe" (i.e., it cannot be lost) whenever either its owner or a contributor storing it is able to 


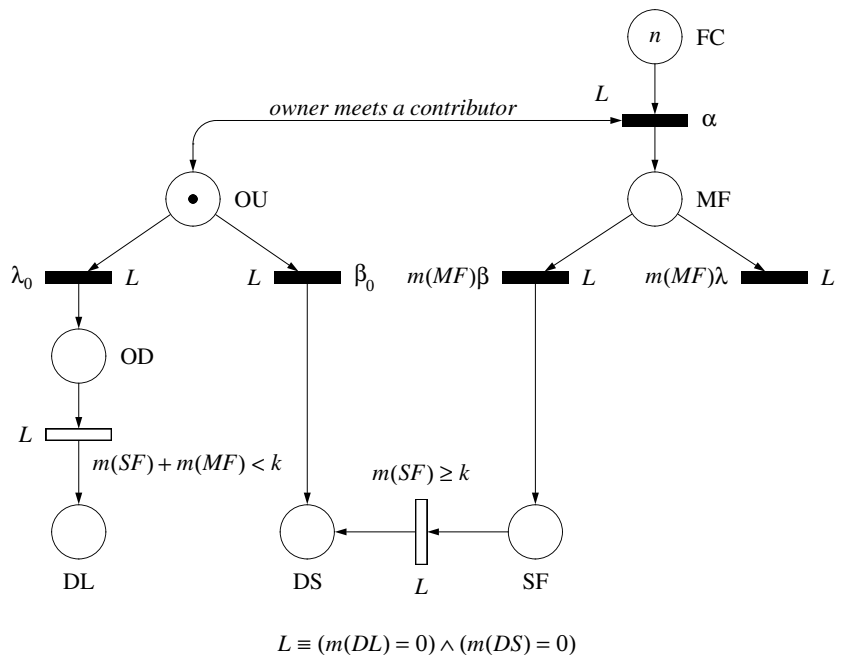

Figure 1. Petri net of the replication and scattering process for an $(n, k)$ erasure code.

access the Internet. In other words, the Internet-based store of our cooperative backup service is abstracted as a "reliable store". Conversely, if a participating device fails before reaching the Internet, then all the fragments it holds are considered lost. Thus, with $(n, k)$ erasure coding, a data item is definitely lost if and only if its owner device fails and less than $k$ contributors hold or have held a fragment of the data item.

Our model consists of three main processes represented by timed transitions with constant rate exponential distributions:

- A process with rate $\alpha$ that models the encounter of a contributor by the data owner, where the owner sends one data fragment to the contributor.

- A process that models the connection of a device to the Internet, with rate $\beta_{0}$ for the owner and $\beta$ for contributors.

- A process that represents the failure of a device, with rate $\lambda_{0}$ for the owner and $\lambda$ for contributors.

The GSPN in Figure 1 is divided into two interacting subnets. The subnet on the left describes the evolution of a data item at the owner device: either it is lost (with rate $\lambda_{0}$ ), or it reaches the Internet store (with rate $\beta_{0}$ ). Places OU and OD denote situations where the owner device is "up" or "down", respectively. The subnet on the right describes: (i) the data replication process leading to the creation of "mobile fragments" (place MF) on contributor devices as they are encountered (with rate $\alpha$ ), and (ii) the processes leading to the storage of the fragments (place $\mathrm{SF}$ ) in the reliable store (rate $\beta$ ), or its loss caused by the failure of the contributor device (rate $\lambda$ ). The initial marking of place FC denotes the number of fragments to create. The transition rates associated with the loss of a data fragment or its storage on the Internet are weighted by the marking of place MF, i.e., the number of fragments that can enable the corresponding transitions.

Two places with associated immediate transitions are used in the GSPN to identify when the data item is safely stored in the reliable store (place DS), or is definitely lost (place DL), respectively. The "data safe" state is reached (i.e., DS is marked) when the original data item from the owner node or at least $k$ fragments from the contributors reach the Internet. The "data loss" state is reached (i.e., DL is marked) when the data item from the owner node is lost and less than $k$ fragments are available. This condition is represented by a predicate associated with the immediate transition that leads to DL. Finally, $L$ is the GPSN "liveliness predicate", true if and only if $m(D S)=m(D L)=0$ : as soon as either DS or DL contains a token, no transition can be fired.

The GSPN model of Figure 1 is generic and can be used to automatically generate the Markov chain associated with any $(n, k)$ erasure code. Examples of Markov chains for different $(\mathrm{n}, \mathrm{k})$ may be found in [4]. The total number of states in such an $(n, k)$ Markov chain is $O\left(n^{2}\right)$. The models we are considering, with reasonably small values of $n$ are tractable using available modeling tools.

\subsection{Quantitative Measures}

We analyze the dependability of our backup service via the probability of data loss, i.e., the asymptotic probability, noted $P L$, of reaching the "data lost" state. For a given erasure code $(n, k), P L$ can be easily evaluated from the corresponding Markov chain using well-known techniques for absorbing Markov chains [11]. The smaller $P L$ is, the more dependable is the data backup service.

To measure the dependability improvement offered by MoSAIC, we compare $P L$ with the probability of data loss $P L_{r e f}$ of a comparable, non-MoSAIC scenario where:

- devices do not cooperate;

- data owner devices fail with rate $\lambda_{0}$;

- data owners gain Internet access and send their data items to a reliable store with rate $\beta_{0}$.

This scenario is modeled by a simple Markov chain where the owner's device can either fail and lose the data or reach the Internet and save the data. The probability of loss in this scenario is: $P L_{r e f}=\frac{\lambda_{0}}{\lambda_{0}+\beta_{0}}$.

We note $L R F$ the data loss probability reduction factor offered by MoSAIC compared to the above non-MoSAIC scenario, where $L R F=P L_{r e f} / P L$. The higher $L R F$, the more MoSAIC improves data dependability. For instance, $L R F=100$ means that data on a mobile device is 
100 times more unlikely to be lost when using MoSAIC than when not using it.

\subsection{Parameters}

$P L$ and $L R F$ depend on a number of parameters $(n, k, \alpha$, $\beta, \lambda, \beta_{0}$, and $\left.\lambda_{0}\right)$. Rather than considering absolute values for the rates of stochastic processes, we consider ratios of rates of pertinent competing processes.

First, the usefulness of cooperative backup will depend on the rates at which contributing devices encounter one another relative to the rate at which connection to the fixed infrastructure is possible. Second, the ef fectiveness of devices towards data backup will depend on the rate at which they fail relative to the rate at which they are able to connect to the Internet to make the data safe. We therefore study $L R F$ as a function of the contributor and data owner effectiveness ratios $\frac{\beta}{\lambda}$ and $\frac{\beta_{0}}{\lambda_{0}}$.

Finally, one may question the assumption that contributors accept all requests, at rate $\alpha$, regardless of their amount of available resources. However, simple backof-the-envelope calculations provide evidence that this is a reasonable assumption. When the replication strategy described in Section 3.1.1 is used, the number of fragments (i.e., storage requests) that a contributor may receive during the time between two consecutive Internet connections is, on average, $\frac{\alpha}{\beta}$. Let $s$ be the size of a fragment: a contributor needs, on average, $V=s\left(\frac{\alpha}{\beta}\right)$ storage units to serve all these requests. If a contributor's storage capacity, $C$, is greater than $V$, it can effectively accept all requests; otherwise, the contributor is saturated and can no longer accept any storage request.

In other words, redefining $\alpha$ as the effective encounter rate (i.e., the rate of encounters of contributors that accept storage requests), and letting $\gamma$ be the actual encounter rate, we have: $\frac{\alpha}{\beta}=\min \left(\frac{\gamma}{\beta}, \frac{C}{s}\right)$. A realistic estimate with $C=2^{30}$ (contributor storage capacity of $1 \mathrm{~GB}$ ) and $s=2^{10}$ (fragment size of $1 \mathrm{~KB}$ ) shows that contributors would only start rejecting requests when $\frac{\gamma}{\beta}>2^{20}$, a ratio that is beyond most realistic scenarios.

\section{Results}

This section discusses the results of our analysis.

\subsection{Overview}

Figure 2 shows the data dependability improvement yielded by MoSAIC with a $(2,1)$ erasure code using the replication strategy outlined in Section 3.1.1. Here, we assume that contributors and owners behave identically, i.e., $\beta_{0}=\beta$ and $\lambda_{0}=\lambda$.

Three observations can be made from this plot. First, as expected, the cooperative backup approach is

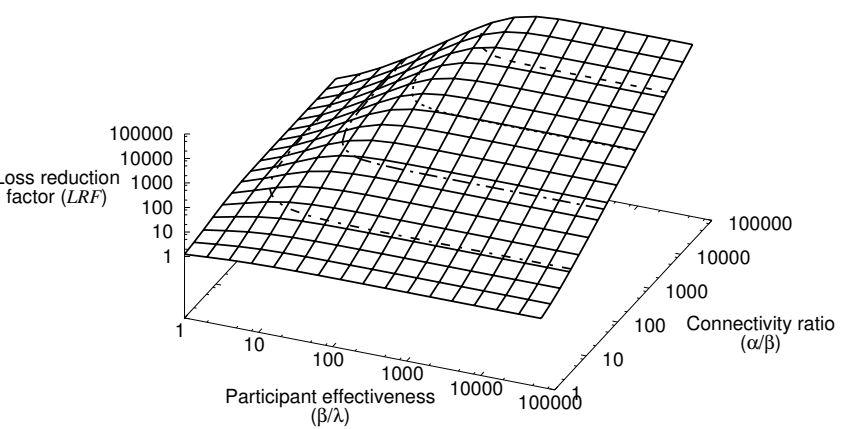

Figure 2. Loss reduction factor $L R F$ for a $(2,1)$ erasure code.

not very relevant compared to the reference backup approach when $\frac{\alpha}{\beta}=1$ (i.e., when Internet access is as frequent as ad hoc encounters). Looking at the contour lines of $L R F$ from Figure 2, it appears that, for the cooperative backup approach to offer at least an order of magnitude improvement over the reference backup scheme, the environment must satisfy $\frac{\beta}{\lambda}>2$ and $\frac{\alpha}{\beta}>10$.

Second, for any given $\frac{\alpha}{\beta}, L R F$ reaches an asymptote after a certain $\frac{\beta}{\lambda}$ threshold. Thus, for any given connectivity ratio $\frac{\alpha}{\beta}$, increasing the infrastructure connectivity to failure rate ratio $\frac{\beta}{\lambda}$ is only beneficial up to that threshold.

The third observation that can be made is that the dependability improvement factor first increases proportionally to $\frac{\alpha}{\beta}$, and then, at a certain threshold, rounds off towards an asymptote (visible on Figure 2 for small values of $\frac{\beta}{\lambda}$ but hidden for high values due to choice of scale). Other $(n, k)$ plots have a similar shape.

\subsection{Asymptotic Behavior}

Figure 3 shows $L R F$ as a function of $\frac{\beta}{\lambda}$, for different values of $\frac{\alpha}{\beta}$ and different erasure codes (again, assuming the data owner's failure and connection rates are the same as those of contributors). This again shows that the maximum value of $L R F$ for any erasure code, as $\frac{\beta}{\lambda}$ tends to infinity, is a function of $\frac{\alpha}{\beta}$. We verified the following formula for a series of codes with $n \in\{2,3,4,5\}$ and $k \in\{1,2,3\}$ (with $k \leq n$ ) and postulate that it is true for all positive values of $n$ and $k$ such that $n \geq k$ :

$$
\lim _{\frac{\beta}{\lambda} \rightarrow \infty}\left(L R F_{n, k}\left(\frac{\alpha}{\beta}, \frac{\beta}{\lambda}\right)\right)=\frac{1}{1-\left(\frac{\frac{\alpha}{\beta}}{1+\frac{\alpha}{\beta}}\right)^{k}}
$$

First, it describes an asymptotic behavior, which confirms our initial numerical observation. Second, it does not depend on $n$. This observation provides useful insight 
in how to choose the most appropriate erasure coding parameters, as we will see in Section 4.3.

We can similarly compute the limiting value of $\operatorname{LRF}(n, k)$ as $\frac{\alpha}{\beta}$ tends to infinity:

$$
\lim _{\frac{\alpha}{\beta} \rightarrow \infty}\left(L R F_{n, k}\left(\frac{\alpha}{\beta}, \frac{\beta}{\lambda}\right)\right)=\frac{\left(1+\frac{\beta}{\lambda}\right)^{n}}{\sum_{x=0}^{k-1}\left(\begin{array}{c}
n \\
x
\end{array}\right)\left(\frac{\beta}{\lambda}\right)^{x}}
$$

This shows that, when $\frac{\alpha}{\beta}$ grows, $L R F$ reaches an asymptote that depends on $\frac{\beta}{\lambda}$.

\subsection{Erasure Coding vs. Simple Replication}

Figure 3 allows us to compare the improvement factor yielded by MoSAIC when different erasure codes are used. The erasure codes shown on the plot all incur the same storage cost: $\frac{n}{k}=2$. In all cases, the maximum dependability improvement decreases as $k$ increases. This is confirmed analytically by computing the following ratio, for any $p>1$ such that $p k$ and $p n$ are integers:

$$
R_{p}=\frac{\lim _{\frac{\beta}{\lambda} \rightarrow \infty}\left(L R F_{p n, p k}\left(\frac{\alpha}{\beta}, \frac{\beta}{\lambda}\right)\right)}{\lim _{\frac{\beta}{\lambda} \rightarrow \infty}\left(L R F_{n, k}\left(\frac{\alpha}{\beta}, \frac{\beta}{\lambda}\right)\right)}=\frac{1-\left(\frac{\frac{\alpha}{\beta}}{1+\frac{\alpha}{\beta}}\right)^{k}}{1-\left(\frac{\frac{\alpha}{\beta}}{1+\frac{\alpha}{\beta}}\right)^{k p}}
$$

We see that $R_{p}<1$ for $p>1$. Thus, we conclude that, from the dependability viewpoint, simple replication (i.e., with $k=1$ ) is always preferable to erasure coding (i.e., with $k>1$ ) above a certain $\frac{\beta}{\lambda}$ threshold. Below that threshold, erasure coding is sometimes preferable to simple replication. Figure 4 compares the dependability improvement yielded by several erasure codes having the same storage cost; only the top-most erasure code (i.e., the surface with the highest $L R F)$ is visible from above. The $(2,1)$ plot is above all other plots, except in a small region where the other erasure codes (thin dashed and dotted lines) yield a higher $L R F$.

A look at a projection of this $3 \mathrm{D}$ plot on the $\frac{\beta}{\lambda}$ and $\frac{\alpha}{\beta}$ plane (omitted for reasons of space), allows the visualization of the region where erasure codes perform better than simple replication. Erasure codes yield a higher data dependability than simple replication in the region defined (roughly) by $\frac{\alpha}{\beta}>100$ and $1<\frac{\beta}{\lambda}<100$. However, in this region, the dependability yielded by erasure codes is typically less than an order of magnitude higher than that yielded by simple replication, even for the (extreme) case where $\frac{\alpha}{\beta}=1000$ (see Figure 3).

Interestingly, similar plots obtained for larger values of $\frac{n}{k}$ (omitted for reasons of space) show that the region where erasure codes prevail tends to shift towards lower

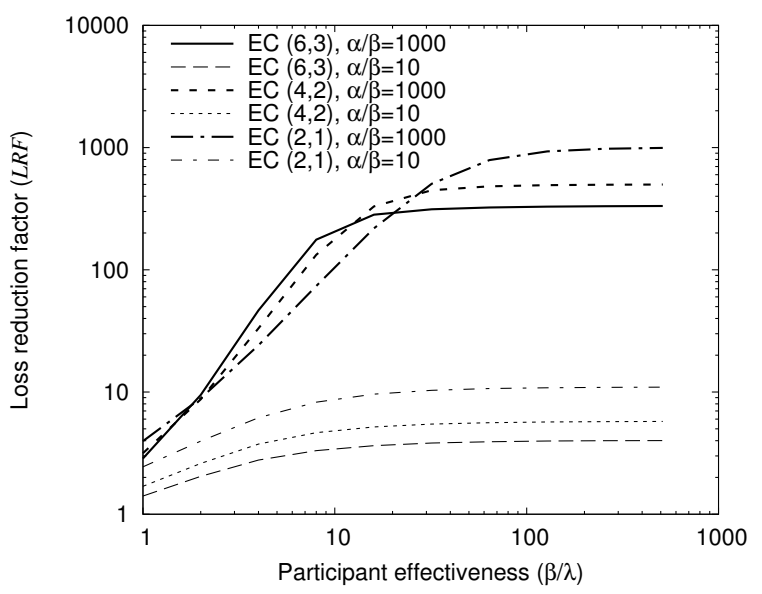

Figure 3. Loss reduction factor for different erasure codes.

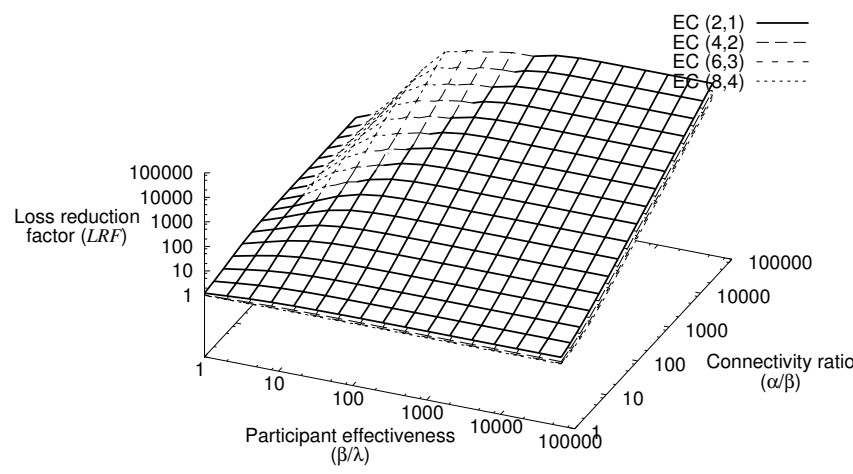

Figure 4. Comparing $L R F$ for different erasure codes with $\frac{n}{k}=2$.

$\frac{\beta}{\lambda}$ values as $\frac{n}{k}$ increases. In other words, the spectrum of scenarios where erasure codes provide better dependability than simple replication narrows as the chosen storage overhead (the $\frac{n}{k}$ ratio) increases.

Nevertheless, when confidentiality is an important criterion, using erasure coding instead of simple replication is relevant. Erasure coding can achieve better confidentiality than simple replication [8] at the cost of a slightly lower asymptotic dependability improvement factor. For instance, in the context of Figure 3, if the user wants to maximize confidentiality while requiring a minimum improvement factor of 100 , a $(6,3)$ erasure code would be chosen rather than simple replication. 


\section{Related Work}

There is a large literature dealing with the design of peerto-peer file sharing architectures and cooperative backup services in mobile and fixed infrastructures. A recent survey can be found in [13]. Here, we focus on related work dealing with the dependability evaluation of replication mechanisms, notably erasure coding algorithms.

Several papers analyze data dependability in distributed and peer-to-peer storage systems. The authors of OceanStore conducted an analytical evaluation of the MTTF (mean time to failure) of a distributed, self-repairing storage system [21]. They conclude that erasure codes yield MTTF orders of magnitude higher than simple replication; however, their computations are based on probability distributions of hard disk failures, which may be quite different from that of individual untrusted peers on the Internet. A similar comparison for peer-topeer storage is proposed in [20], using a stochastic model. They conclude on the unsuitability of erasure codes in a peer-to-peer environment where peer availability is low. The major difference between these studies and our work is that the authors model a data block repair process that is inexistent in the context of a mostly-disconnected peer-to-peer backup system, notably because data owners cannot be made aware of contributor failures.

In [15], the authors analyze erasure code replication and compare the resulting data availability as a function of individual host availability (assuming each host stores exactly one fragment of the original data) and erasure code parameters $(n, k)$. They identify a "switch point" between scenarios where erasure coding is preferable (from a data availability viewpoint) and scenarios where simple replication should be used. More precisely, they conclude that simple replication yields better data availability when host availability is low.

Our results comparing erasure codes and simple replication in terms of dependability are in agreement with those obtained on simpler models $[1,15,20]$. We observe a switch point similar to that of [15]. For instance, in our model, whether erasure codes yield better data dependability than simple replication depends on $\frac{\alpha}{\beta}$ and $\frac{\beta}{\lambda}$ (see, e.g., Figure 4).

Building on a similar analysis, TotalRecall [1], a peer-to-peer storage system, proposes mechanisms to automate availability management, which includes dynamic parameterization of erasure coding replication based on predicted host availability. However, the authors do not consider the use of erasure codes as a means to improve data confidentiality [8]. Additionally, the mobile environment we are addressing leads to a wider range of scenarios (and connectivity). A dynamic replication strategy for peer-to-peer cooperative data storage among untrusted nodes is also presented in [19], though they do not consider the use of erasure codes.

\section{Conclusion and Future Work}

Our evaluation methodology allowed us to achieve our goals (see Section 1). First, we have a better understanding of the scenarios where MoSAIC yields noticeable data dependability improvement. Namely, we showed that the cooperative backup approach is beneficial (i.e., yields data dependability an order of magnitude higher than without MoSAIC) only when $\frac{\beta}{\lambda}>2$ and $\frac{\alpha}{\beta}>10$. We demonstrated that MoSAIC can decrease the probability of data loss by a factor that can be as large as the ad hoc to Internet connectivity ratio $\frac{\alpha}{\beta}$.

Second, we compared simple replication and erasure codes and concluded that erasure codes provide an advantage (dependability-wise) over simple replication only in narrow scenarios. Those scenarios are restricted to low $\frac{\beta}{\lambda}$ and high $\frac{\alpha}{\beta}$ and the dependability improvement provided by erasure codes in those cases is typically less than an order of magnitude. Measurements of actual use cases are needed in order to see what real-world situations these scenarios map to.

Based on our results, several replication strategies can be envisioned. One possible strategy would be to maximize data dependability for a given user-specified storage overhead. Since in most scenarios little can be gained from using erasure codes, and since the consequence of a wrong decision would be detrimental to data dependability (e.g., choosing erasure coding in a scenario where simple replication would have been more beneficial), the best way to maximize data dependability is to always use simple replication.

Other replication strategies can be imagined. Instead of focusing only on dependability, users may specify additional fragmentation to increase confidentiality [8]. Such a strategy could maximize fragmentation (i.e., by choosing a high $k$ value) according to environmental parameters, while honoring a user-specified minimum dependability improvement factor. The ad hoc and Internet connectivity rates could be estimated, for instance, by collecting actual data about single-hop device encounters of a device that is carried around according to some mobility scenario. These environmental parameters as well as the effectiveness of encountered contributors could also be estimated on-line based on past observations, perhaps augmented by user input, and used a hint to the replication strategy.

Current and future work also includes refining our model to allow for the distribution of more than one fragment per contributor. Doing so would allow the evaluation of a wider range of replication and dissemina- 
tion strategies. So-called rate-less erasure codes allow the production of an unlimited number of distinct fragments, out of which any $k$ suffice to recover the original data [17]. Their use could also be evaluated with little impact on our model, for instance by choosing higher values of parameter $n$.

\section{Acknowledgements}

We thank our colleagues Thomas Robert and Benjamin Lussier for their insightful and constructive comments on an earlier version of this paper.

\section{References}

[1] R. Bhagwan, K. Tati, Y-C. Cheng, S. Savage, G. M. VOELKER. Total Recall: System Support for Automated Availability Management. In Proc. of the ACM/USENIX NSDI, March 2004.

[2] M. Boulkenafed, V. Issarny. AdHocFS: Sharing Files in WLANs. In Proc. of the 2nd Int. Symp. on Network Computing and Applications, April 2003.

[3] L. Courtès, M-O. Killujian, D. Powell. Storage Tradeoffs in a Collaborative Backup Service for Mobile Devices. In Proc. of the 6th European Dependable Computing Conf., pp. 129-138, IEEE CS Press, October 2006.

[4] L. CourTès. Cooperative Data Backup for Mobile Devices, PhD Thesis, LAAS-CNRS, Université de Toulouse, November 2007.

[5] L. Courtès, M-O. Killijian, D. Powell. Security Rationale for a Cooperative Backup Service for Mobile Devices. In Proc. of the Latin-American Symp. on Dependable Computing, Springer-Verlag, 2007.

[6] D.R Cox, H.D. MiLler. The Theory of Stochastic Processes. Chapman and Hall Ltd., 1965.

[7] L. P. Cox, C. D. Murray, B. D. Noble. Pastiche: Making Backup Cheap and Easy. In 5th USENIX OSDI, pp. 285-298, December 2002.

[8] Y. Deswarte, L. Blain, J-C. Fabre. Intrusion Tolerance in Distributed Computing Systems. In Proc. of the IEEE Symp. on Research in Security and Privacy, pp. 110-121, May 1991.

[9] G. R. Ganger, P. K. Khosla, M. Bakkaloglu, M. W. Bigrigg, G. R. Goodson, S. Oguz, V. Pandurangan, C. A. N. Soules, J. D. Strunk, J. J. Wylie. Survivable Storage Systems. In Proc. of the DARPA Information Survivability Conf. \& Exposition (DISCEX), pp. 184-195, IEEE CS Press, June 2001.

[10] A. KarYPIDIS, S. Lalis. OmniStore: A System for Ubiquitous Personal Storage Management. In Proc. of the Annual IEEE Int. Conf. on Pervasive Computing and Communications (PerCom), pp. 136-147, IEEE CS Press, March 2006.

[11] J. G. Kemeny, J. L. Snell. Finite Markov Chains. D. Van Nostrand Co., Inc., Princeton, New Jersey, USA, 1960.

[12] M-O. Killijian, D. Powell, M. BanÂtre, P. Couderc, Y. RoudiER. Collaborative Backup for Dependable Mobile
Applications. In Proc. of 2nd Int. Workshop on Middleware for Pervasive and Ad-Hoc Computing (Middleware 2004), pp. 146-149, ACM Press, October 2004.

[13] M-O. Killijan, L. Courtès, D. Powell. A Survey of Cooperative Backup Mechanisms. Technical Report LAAS Report 06472, LAAS-CNRS, October 2006.

[14] J. Kubiatowicz, D. Bindel, Y. Chen, S. Czerwinski, P. Eaton, D. Geels, R. Gummadi, S. Rhea, H. Weatherspoon, W. Weimer, C. Wells, B. Zhao. OceanStore: An Architecture for Global-Scale Persistent Storage. In Proc. of the 9th ASPLOS, pp. 190-201, November 2000.

[15] W. K. Lin, D. M. Chiu, Y. B. LeE. Erasure Code Replication Revisited. In Proc. of the 4th P2P, pp. 90-97, 2004.

[16] M.A. Marsan, G. Balbo, G. Conte, S. Donatelli, G. FranceschinIs. Modeling with Generalized Stochastic Petri Nets. John Wiley \& Sons Ltd., 1995.

[17] M. Mitzenmacher. Digital Fountains: A Survey and Look Forward. In Proc. of the IEEE Information Theory Workshop, pp. 271-276, October 2004.

[18] M. Papadopouli, H. SchulzrinNe. Seven Degrees of Separation in Mobile Ad Hoc Networks. In IEEE Conf. on Global Communications (GLOBECOM), November 2000.

[19] K. Ranganathan, A. Iamnitchi, I. Foster. Improving Data Availability Through Dynamic Model-Driven Replication in Large Peer-to-Peer Communities. In Proc. of the Workshop on Global and Peer-to-Peer Computing on Large Scale Distributed Systems, pp. 376-381, IEEE CS Press, May 2002.

[20] A. Vernois, G. Utard. Data Durability in Peer to Peer Storage Systems. In Proc. of the 4th Workshop on Global and Peer to Peer Computing, pp. 90-97, IEEE CS Press, April 2004.

[21] H. Weatherspoon, J. Kubiatowicz. Erasure Coding vs. Replication: A Quantitative Comparison. In Revised Papers from the 1st Int. Workshop on Peer-to-Peer Systems, pp. 328-338, Springer-Verlag, 2002.

[22] L. Xu, V. Bohossian, J. Bruck, D. G. Wagner. Low Density MDS Codes and Factors of Complete Graphs. In IEEE Transactions on Information Theory, 45(1), November 1999, pp. 1817-1826.

[23] L. Xu. Hydra: A Platform for Survivable and Secure Data Storage Systems. In Proc. of the ACM Workshop on Storage Security and Survivability, pp. 108-114, ACM Press, November 2005.

[24] L. Yin, G. CaO. Supporting Cooperative Caching in Ad Hoc Networks. In IEEE Transactions on Mobile Computing, 5(1), January 2006, pp. 77-89.

[25] Z. ZHANG. Routing in Intermittently Connected Mobile Ad Hoc Networks and Delay Tolerant Networks: Overview and Challenges. In IEEE Communications Surveys \& Tutorials, 8, January 2006, pp. 24-37. 